\title{
Rapid screening for chromosomal aneuploidies using array-MLPA
}

\author{
Jing-Bin Yan ${ }^{1,2}$, Miao Xu', Can Xiong ${ }^{1}$, Da-Wen Zhou', Zhao-Rui Ren'1, Ying Huang ${ }^{1,2}$, Monique Mommersteeg ${ }^{3}$, \\ Rinie van Beuningen ${ }^{3}$, Ying-Tai Wang ${ }^{4}$, Shi-Xiu Liao ${ }^{4}$, Fanyi Zeng ${ }^{1,2,5}$, Ying Wu ${ }^{1,3^{*}}$ and Yi-Tao Zeng ${ }^{1,2^{*}}$
}

\begin{abstract}
Background: Chromosome abnormalities, especially trisomy of chromosome 21, 13, or 18 as well as sex chromosome aneuploidy, are a well-established cause of pregnancy loss. Cultured cell karyotype analysis and FISH have been considered reliable detectors of fetal abnormality. However, results are usually not available for 3-4 days or more. Multiplex ligation-dependent probe amplification (MLPA) has emerged as an alternative rapid technique for detection of chromosome aneuploidies. However, conventional MLPA does not allow for relative quantification of more than 50 different target sequences in one reaction and does not detect mosaic trisomy. A multiplexed MLPA with more sensitive detection would be useful for fetal genetic screening.
\end{abstract}

Methods: We developed a method of array-based MLPA to rapidly screen for common aneuploidies. We designed 116 universal tag-probes covering chromosomes $13,18,21, X$, and $Y$, and 8 control autosomal genes. We performed MLPA and hybridized the products on a 4-well flow-through microarray system. We determined chromosome copy numbers by analyzing the relative signals of the chromosome-specific probes.

Results: In a blind study of 161 peripheral blood and 12 amniotic fluid samples previously karyotyped, 169 of 173 (97.7\%) including all the amniotic fluid samples were correctly identified by array-MLPA. Furthermore, we detected two chromosome $X$ monosomy mosaic cases in which the mosaism rates estimated by array-MLPA were basically consistent with the results from karyotyping. Additionally, we identified five $Y$ chromosome abnormalities in which G-banding could not distinguish their origins for four of the five cases.

Conclusions: Our study demonstrates the successful application and strong potential of array-MLPA in clinical diagnosis and prenatal testing for rapid and sensitive chromosomal aneuploidy screening. Furthermore, we have developed a simple and rapid procedure for screening copy numbers on chromosomes 13, 18, 21, $X$, and $Y$ using array-MLPA.

\section{Background}

Chromosome abnormalities are a well-established cause of pregnancy loss. The most common are autosomal aneuploidy $(\sim 75 \%)$, followed by polyploidy $(\sim 13 \%)$, sex chromosome abnormalities $(\sim 8 \%)$ and structural imbalance $(\sim 4 \%)[1,2]$. Trisomy of chromosome 21,13 , or 18 as well as sex chromosome aneuploidy account for 60 $80 \%$ of abnormal fetal karyotypes detected in cultured amniotic fluid cells [3].

For non-mosaic standard trisomy, cultured karyotype analysis has been considered a reliable detector of fetal

\footnotetext{
* Correspondence: ying.wu@philips.com; ytzeng@stn.sh.cn

'Institute of Medical Genetics, Children's Hospital of Shanghai, Shanghai Jiao Tong University, Shanghai, P.R. China

Full list of author information is available at the end of the article
}

abnormality [4]. However, the sensitivity of karyotyping depends on the number of cells established in a particular culture, and results are usually not available for 3-4 days or more. Furthermore, it is very difficult to identify chromosome microdeletions. In addition to karyotype analysis, fluorescence in situ hybridization (FISH), an easy-to-handle, rapid, and highly sensitive tool for genetic analysis, has been developed in the past two decades [5-14]. Recently, AneuVysion FISH analysis has become the most common rapid screening method for prenatal or neonatal aneuploidies in a clinical setting. In most laboratories, AneuVysion analysis for prenatal testing costs between $\$ 300$ and $\$ 350$. However, FISH is labor-intensive and not easily applicable to a large number of samples in clinical diagnostic settings.

\section{Ciomed Central}


Multiplex quantitative fluorescence PCR (QF-PCR) provides the possibility to detect copy number variation of chromosomal sequences in several hours [12,15-19]. It also has the advantage of being much cheaper and allowing the simultaneous processing of larger numbers of samples than FISH and karyotyping analysis. However, the presence of multiple primer pairs in a PCR reaction reduces the reliability of the quantification. To solve these technical problems, multiplex ligation-dependent probe amplification (MLPA) has emerged as an alternative to standard PCR-based techniques for detection of the chromosome aneuploidies [20-22]. It allows for relative quantification of up to 50 different target sequences in one reaction and does not require living cells or cell culture. It is less labor-intensive and less expensive compared to karyotyping and FISH. Therefore, MLPA has been widely applied for molecular diagnosis of genetic diseases such as DMD, Spinocerebellar ataxia type 15 and chromosomal aneuploidies [21,23-26]. Moreover, a commercial MLPA kit based on length discrimination of the ligation products for detection of aneuploidies on chromosomes 13, 18, 21, $\mathrm{X}$ and $\mathrm{Y}$ has been developed. Its turn-around time can be as rapid as 30 hours.

A primary drawback of MLPA is its dependence on length-based discrimination of the ligation products. To differentiate between amplification products, the probes contain a non-hybridizing stuffer sequence of variable length. Therefore, MLPA limits the number of probes to 50 pairs or fewer. The size differences complicate the essential quantitative PCR step, since smaller fragments are amplified more efficiently. To resolve these technical hurdles, we have recently developed a universal flowthrough array to quantify the MLPA amplification products, and successfully applied the array-MLPA assay for detection of deletions and duplications in Duchenne muscular dystrophy patients [27].

To determine whether the array-MLPA format can be used in clinical diagnostic settings to screen for common aneuploidies, we performed array-MLPA analysis on 161 peripheral blood and 12 amniotic fluid samples. Moreover, we confirmed the genotypes obtained on array-MLPA by G-banded karyotype analysis.

\section{Methods \\ Patient materials}

The peripheral blood samples were collected from 76 unrelated patients and 85 healthy individuals from Shanghai Children's Hospital. Twelve amniotic fluid samples (15-20 ml/sample) without blood contamination were obtained from the Institute of Medical Genetics in Henan Provincial People's Hospital. The amniotic fluid samples were collected from cases with abnormal maternal serum screening results. Informed consent was obtained from each participant. The study was approved by the ethics committee of Shanghai Children's Hospital.

Genomic DNA was extracted from peripheral blood or amniotic fluid cells ( $5 \mathrm{ml} / \mathrm{sample}$ ) using the phenol/ chloroform method. The concentration and quality of the DNA was estimated by measuring the optical density (OD) at $260 \mathrm{~nm}$ and $280 \mathrm{~nm}$ using a spectrophotometer (DU800, Beckman, US). Each sample was checked on gel electrophoresis. A DNA sample was discarded for further analyses if OD 260/280 ratio was not within the normal range (1.8-2.0). Additionally, the concentration of each DNA sample was standardized to 100 $\mathrm{ng} / \mu \mathrm{L}$. The data processing of the array-MLPA tests were performed without the knowledge of karyotype results.

\section{MLPA probe design and reaction}

The design of the assay probes was essentially as described for array-MLPA [27]. The target sequences on chromosomes 13, 18, 21, X and Y were designed using the Ensembl genome browser (http://www.ensembl.org). Additionally, the target sequences of 8 autosomal genes on chromosomes $2,4,5,11,12$, and 15 were designed as control probes. Ultimately, 116 pairs of MLPA probes covering chromosomes 13 (probe $\mathrm{n}=28$ ), 18 (probe $\mathrm{n}=$ 26), 21 (probe $n=22), X$ (probe $n=22), Y$ (probe $n=$ 10) and 8 control probes were included in the study. The accession numbers and probe sequences are shown in Additional file 1, Table S1.

All of the oligonucleotides were chemically synthesized (Illumina, CA, USA) in a salt-free environment (25 nmol scale) and used without further purification. The probe mix was prepared at a final concentration of 4 nM per probe. MLPA reagents were purchased from MRC-Holland (Amsterdam, The Netherlands). The MLPA reaction was performed using a standard protocol described previously [27].

\section{Universal flow-through array and hybridization}

In this study, a 4-well universal flow-through array with 124 pairs of 20-mer oligonucleotide tag-probes was used (PamGene, Den Bosch, The Netherlands). The preparation of the array using a porous aluminum-oxide substrate was performed as previously described [27-29]. In each of the arrays up to 400 probes can be spotted on the substrate. In comparison with two-dimensional geometry, the reactive surface in the porous substrate is increased approximately 500-fold. During hybridization, samples are actively pumped back and forth through the porous structure, resulting in a hybridization time of 530 minutes. Schematic depiction of the array technology is shown in Additional file 2, Figure S1.

The array hybridization was performed using a standard protocol described previously [27]. A single chip 
contained 4 identical arrays. One normal control and three other samples were analyzed in parallel. After washing the arrays, images were recorded at $500 \mathrm{~ms}$, $1000 \mathrm{~ms}, 1500 \mathrm{~ms}$ and $3000 \mathrm{~ms}$ exposure time using a Cy5 filter set. Additionally, three independent experiments for each of 30 normal control individuals (15 males and 15 females) were performed to determine the accuracy of array-MLPA and the distributions of the normal copy numbers.

\section{Data analysis}

Each array image was converted into spot intensity values using BioNavigator software (PamGene, Den Bosch, The Netherlands). Pixel-by-pixel cross-correlation was used to segment signal, background and artifact pixel populations. Median signal intensities were obtained for each spot on the array and local background was subtracted. A cutoff value for a positive signal was defined as three times above the standard deviation (SD) of the background. The mean signal of duplicate spots on each array was normalized using the mean signal of the eight control autosomal genes. Copy numbers of chromosomes 13, 18, 21, X and Y were computed as the ratio of test signal to autosomal control signal for each sample. The copy number data were exported into Microsoft Excel (Microsoft, Redmond, WA), where the median, SD and coefficients of variation (CV) of these chromosomes were calculated.

Normal copy number values for chromosomes 13, 18, 21 and $\mathrm{X}$ were defined between 0.85 and 1.15 in normal females, whereas the normal values of chromosomes $\mathrm{X}$ and $\mathrm{Y}$ were defined between 0.35 and 0.65 in normal males. Chromosomal trisomy was considered if the copy number was measured as 1.35 to 1.65 in a sample. The XXY type was detected if the copy number of chromosome $\mathrm{X}$ ranged from 0.85 to 1.15 . When the ratio was below 0.65 in female patients, Turner's syndrome was identified. Samples that showed an average CV above $15 \%$ were considered to be unreliable.

\section{Karyotype analysis}

Peripheral blood cells or amniotic fluid cells (10-15 ml/ sample) were cultured according to the standard techniques on culture slides followed the method described by
ISCN [28]. G banding was used for analysis and more than 20 metaphase chromosomes were routinely investigated. The results derived from array-MLPA and karyotyping were compared.

\section{Deletion confirmation}

Y-chromosome-specific PCRs for SRY, TSPY, RBMY genes were performed to confirm deletions on the mosaic samples detected by array-MLPA. The primer sequences are listed in Table 1 . The PCR reaction conditions were $95^{\circ} \mathrm{C}, 5 \mathrm{~min}$ followed by 33 cycles of $95^{\circ} \mathrm{C}$, $1 \mathrm{~min}, 60^{\circ} \mathrm{C}, 1 \mathrm{~min}\left(55^{\circ} \mathrm{C}\right.$ for TSPY gene) and $72^{\circ} \mathrm{C}, 2$ min along with final extension at $72^{\circ} \mathrm{C}$ for $10 \mathrm{~min}$. The PCR product was then subjected to $1 \%$ agarose gel for electrophoretic separation.

\section{Results}

Array-MLPA is not a length-based discrimination method, which can overcome the main drawback of MLPA. The sensitivity and specificity of array-MLPA is based on the universal flow-through array hybridization and data analysis. To determine the reliability of arrayMLPA, 30 normal control individuals (15 males and 15 females) were first tested with replicate measurements. The mean standard deviation across the 30 control individuals was 0.024 , indicating the low inter-individual variability on the array-MLPA test. All probes designed on chromosomes 13, 18, 21 and $\mathrm{X}$ of female samples had an average relative signal of about 1.0, whereas the average signal of chromosomes $\mathrm{X}$ and $\mathrm{Y}$ in males was approximately 0.5 . The copy numbers of almost all the controls were located within the normal distributions ( two grey lines in Figure 1). The intra-assay standard deviations of the probe signals on chromosome 13, 18, $21, \mathrm{X}$ and $\mathrm{Y}$ ranged from 0.018 to 0.031 . Examples of the copy numbers on array-MLPA for a normal female and a normal male are shown in Figure 2. The average gene copy numbers on the five chromosomes in the normal controls are listed in Additional file 3, Table S2. Overall, $89.4 \%$ of the relative probe signals in the normal controls were within the normal range $(0.85$ to 1.15).

Next, array-MLPA was performed on 161 peripheral blood and 12 amniotic fluid samples including the 30

\section{Table 1 PCR primers for deletion confirmation}

\begin{tabular}{llcc}
\hline Gene & Primer sequence & Annealing Temperature $\left({ }^{\circ} \mathbf{C}\right)$ & PCR product length $(\mathbf{b p})$ \\
\hline SRY & 5'-GAGACTCAGACAGCGAAGTA-3' & 60 & 3155 \\
TSPY & 5'-ACGTCCAGGATAGAGTGAAG-3' & 55 & 3396 \\
& 5'-TTACCTCCGTACCATCTACC-3' & & 3174 \\
RBMY & 5'-GAAGTCAGCCTCCAACTAAG-3' & 60 & \\
& 5'-CCGTTATCCTCTTCAGTCAC-3' & & \\
\hline
\end{tabular}




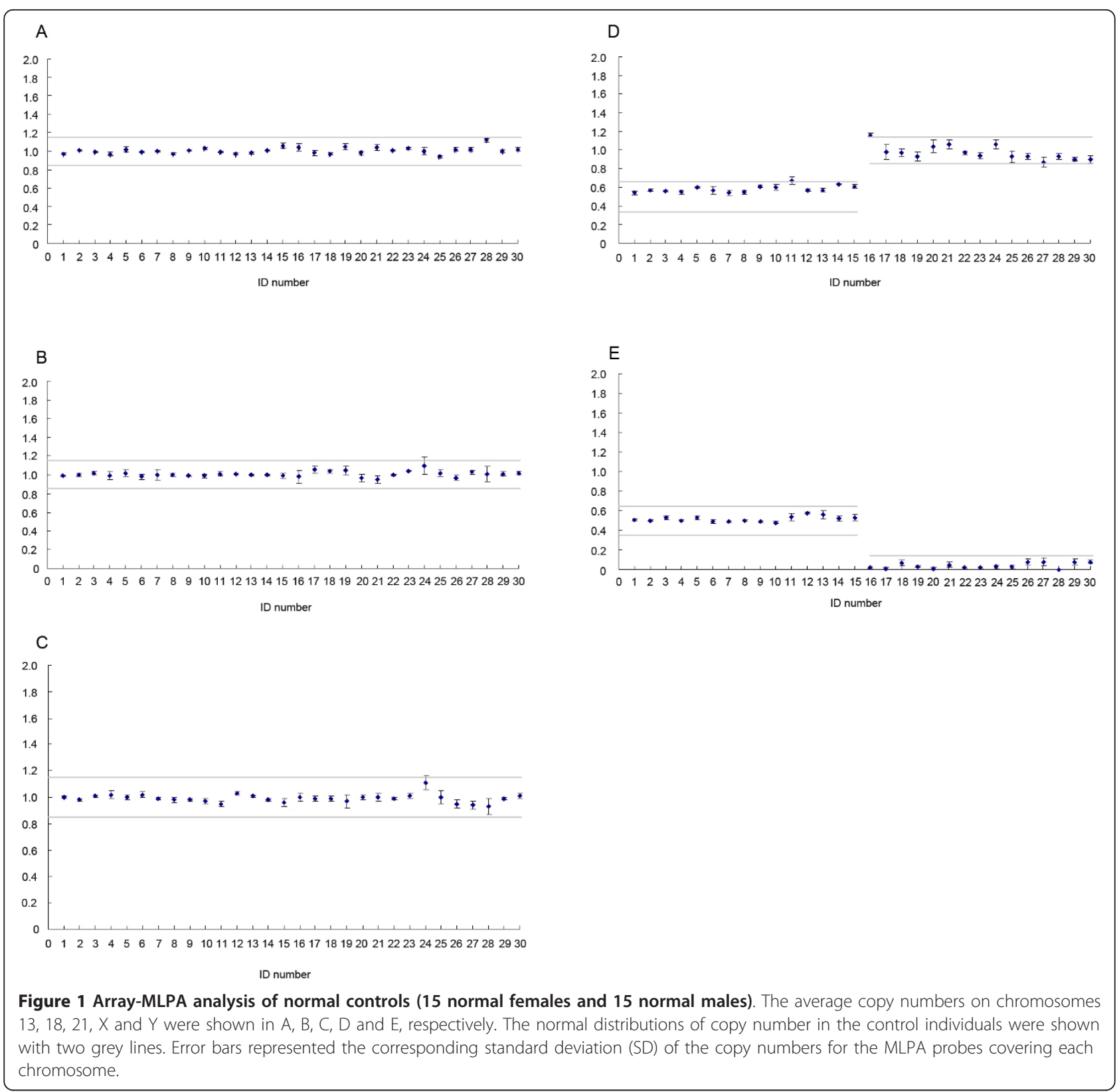

normal controls described above. Karyotype analysis was independently carried out on these samples. The results obtained by array-MLPA and the corresponding Gbanded karyotypes were presented in Table 2. Of 173 tested samples, 169 (97.7\%) including 12 amniotic fluid samples were correctly identified by array-MLPA. Figure 3 showed the abnormal copy numbers on chromosome 21 or X for two fetus samples. One was a male fetus (A9) in which the average copy number of chromosome 21 reached 1.53 indicating trisomy 21 (Figure 3A), while another was a male fetus (A10) in which the copy number on the $\mathrm{X}$ chromosome was $0.85 \pm 0.02$ suggesting XXY (Figure 3B). However, four abnormal karyotypes including 46,XX,-14,+t(14;21);(p11.2;p11.2); 46,XY,rec (14)(18qter $\rightarrow 18 q 22:: 14 q$ ter)pat; 46,XY,inv(9)(p12q21) and $46, \mathrm{XY}, \mathrm{t}(1 ; 7)(\mathrm{p} 36.3 ; \mathrm{p} 13)$ could not be detected by array-MLPA (Table 2). A comparison of the results from array-MLPA and karyotyping was summarized in Table 3.

In this study, two cases (B10 and B11) with chromosome $\mathrm{X}$ monosomy mosaicism were identified successfully by array-MLPA (Table 2). For mosaic samples, the ratio reflects the extent of mosaicism, e.g. ratio 0.75 for a $50 \%$ chromosome $\mathrm{X}$ monosomy in a female sample. The results showed that the average copy numbers of chromosome $\mathrm{X}$ were 0.71 and 0.72 in the two samples. 
A
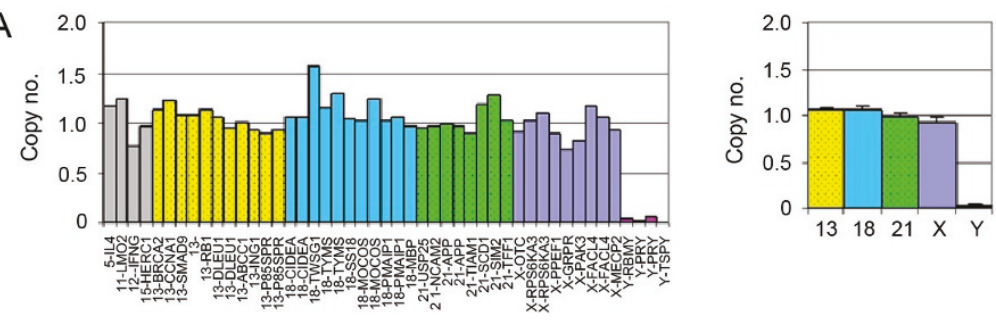

\begin{tabular}{ccc}
\hline Chromosome & Number of probes & N59 \\
13 & 11 & $1.05 \pm 0.03$ \\
18 & 11 & $1.05 \pm 0.05$ \\
21 & 8 & $0.97 \pm 0.05$ \\
$X$ & 9 & $0.93 \pm 0.05$ \\
$Y$ & 4 & $0.03 \pm 0.01$ \\
\hline \multicolumn{2}{c}{ Description } & Normal Female \\
\hline
\end{tabular}

B
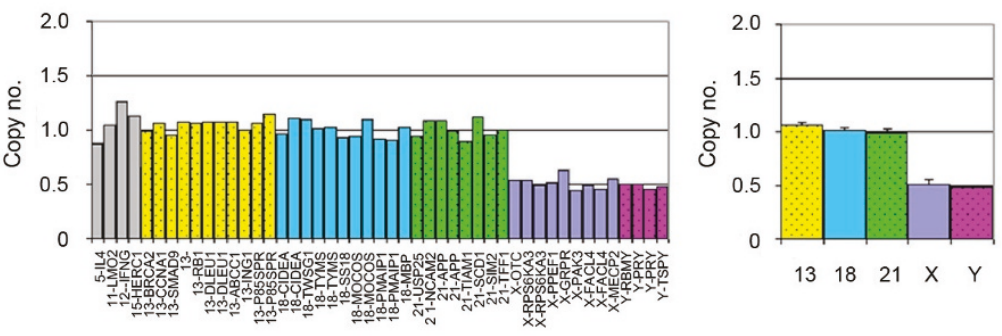

\begin{tabular}{ccc}
\hline Chromosome & Number of probes & $\mathrm{N} 1$ \\
13 & 11 & $1.06 \pm 0.02$ \\
18 & 11 & $1.02 \pm 0.03$ \\
21 & 8 & $0.99 \pm 0.05$ \\
$\mathrm{X}$ & 9 & $0.51 \pm 0.01$ \\
$\mathrm{Y}$ & 4 & $0.49 \pm 0.03$ \\
\hline \multicolumn{3}{c}{ Description } \\
\hline
\end{tabular}

Figure 2 A normal female and normal male were analyzed with array-MLPA. The left figures showed the relative signal of each probe. The probes on chromosomes $13,18,21, \mathrm{X}$ and $\mathrm{Y}$ were depicted in yellow, blue, green, purple and purplish red, respectively. Eight autosomal control probes were shown in grey. The right figures showed the average copy numbers on each chromosome. Error bars represented the

corresponding SD of the copy numbers on the MLPA probes covering each chromosome. The copy number of each chromosome was listed in the lower tables. In the female, the copy numbers on chromosomes 13, 18, 21 and $X$ were defined between 0.85 and 1.15. In the male, the copy numbers on chromosomes 13,18 and 21 were nearly 1 , while the copy numbers on chromosomes $X$ and $Y$ were approximately 0.5 .

This suggested that approximately $50 \%$ of the sample cell population carried chromosome X monosomy. Gbanded karyotype analysis showed that their chromosome karyotypes were 45,X/46,X,i(X)(q10) and 45,X/47, XXX for B10 and B11 respectively, which were basically consistent with array-MLPA results (Figure 4).

Additionally, five cases (B5-B9) with exceptive sex chromosome aneuploidies were identified using the criterion that at least four of ten $\mathrm{Y}$ chromosome-specific probes detected an abnormality. The probe signals in the TSPY gene were identified in all of the five cases (B5-B9). While the signals in the $R B M Y$ gene were detected in three samples (B7-B9). Subsequently, the G- banding analysis revealed that they were chromosome $\mathrm{X}$ monosomy mosaics with chromosome $\mathrm{Y}$ or unknown marker chromosome (mar) (Table 2). Their karyotypes were $45, \mathrm{X} / 45, \mathrm{X}$, mar for samples $\mathrm{B} 5-\mathrm{B} 6,45, \mathrm{X} / 46, \mathrm{XY}$ for the sample B7 and 45,X/46,XY(Yq-) for samples B8-B9, respectively. PCR analysis of the RBMY, SRY and TSPY genes confirmed that the unknown marker chromosome found in samples B5 and B6 was indeed a part of chromosome Y (Figure 5).

\section{Discussion}

Trisomy of chromosome 21,13 , or 18 as well as sex chromosome aneuploidy are the main genetic diseases 
Table 2 Summary of the results of array-MLPA and karyotype analysis

\begin{tabular}{|c|c|c|c|}
\hline Sample ID & Array-MLPA & Karyotype & Sample type \\
\hline N1-N53 & Normal $13,18,21, X, Y$ & $46, X Y$ & \\
\hline N54-N85 & Normal $13,18,21, x$ & $46, X X$ & \\
\hline B1-B4 & Monosomy X & $45, X$ & \\
\hline B5-B6 & Monosomy X+TSPY signals & $45, X / 45, X,+$ mar? & \\
\hline B7 & Monosomy X+RBMY, TSPY signals & $45, X / 46, X Y$ & \\
\hline B8-B9 & Monosomy X+ RBMY, TSPY signals & $45, X / 46, X Y(Y q-?)$ & \\
\hline B10 & Abnormal monosomy $X\left(X_{R}=0.71\right)$ & $45, X / 46, X, i(X)(q 10)$ & \\
\hline B11 & Abnormal monosomy $X\left(X_{R}=0.72\right)$ & $45, X / 47, X X X$ & Blood \\
\hline B12-B29 & Trisomy $21\left(21_{R}=1.36\right.$ to 1.62$)$ & $47, X X,+21$ & \\
\hline B30 & Normal $13,18,21, X, Y$ & $46, X Y$,rec(14)(18qter $\rightarrow 18 q 22:: 14 q$ ter)pat & \\
\hline B31-B72 & Trisomy $21\left(21_{R}=1.37\right.$ to 1.56$)$ & $47, X Y,+21$ & \\
\hline B73 & Male with extra $X$ & $47, X X Y$ & \\
\hline B74 & Normal $13,18,21, x$ & $46, X X,-14,+\mathrm{t}(14 ; 21) ;(\mathrm{p} 11.2 ; \mathrm{p} 11.2)$ & \\
\hline B75 & Normal $13,18,21, X, Y$ & $46, X Y, \operatorname{inv}(9)(p 12 q 21)$ & \\
\hline B76 & Normal $13,18,21, X, Y$ & $46, \mathrm{XY}, \mathrm{t}(1 ; 7)(\mathrm{p} 36.3 ; \mathrm{p} 13)$ & \\
\hline A1-A4 & Trisomy $21\left(21_{R}=1.38\right.$ to 1.52$)$ & $47, X X,+21$ & Amniotic fluid \\
\hline A5-A9 & Trisomy $21\left(21_{R}=1.38\right.$ to 1.53$)$ & $47, X Y,+21$ & \\
\hline A10-A11 & Male with extra $X\left(X_{R}=0.85, Y_{R}=0.51\right)$ & $47, X X Y$ & \\
\hline A12 & Normal $13,18,21, X, Y$ & $46, X Y$ & \\
\hline
\end{tabular}

of newborns. So far, there is no effective treatment for these diseases. Therefore, it is crucial to develop a rapid, reliable and highly sensitive molecular diagnostic technique. In this study, a 4-well universal flow-through array based on a three-dimensional aluminum-oxide substrate was used to analyze MLPA amplification products (array-MLPA). The main advantages of array-MLPA over other techniques such as karyotype analysis, FISH and multiplex PCR, are its simplicity, speed, and analysis of 124 detector probes in parallel. Four different samples for 124 genetic markers can be tested all at once within 6 hours starting from fresh blood or amniotic fluid samples. The cost of the universal chip is limited to $\$ 50$ per sample. An additional advantage of this method is the automated measurement and data analysis. Compared to AneuVysion FISH analysis, the array-MLPA system demonstrates at least 4-fold shorter assay time and at least 6-fold less assay cost. The reproducibility and accuracy of the array technology were demonstrated previously in studies on gene expression profiling $[29,30]$ and quantification of PMP22 gene copy number [31] and detection of DMD genomic rearrangements [27].

To further investigate the applicability of array-MLPA for rapid chromosomal aneuploidy screening, especially in fetuses at risk for Down's syndrome, we performed array-MLPA analysis on 161 peripheral blood and 12 amniotic fluid samples. Our results showed that 169 of $173(97.7 \%)$ samples including all the amniotic fluid samples identified by array-MLPA were concordant with karyotype analysis. Our study demonstrates the successful application and strong potential of array-MLPA in clinical diagnosis and prenatal testing for rapid chromosomal aneuploidy screening. Further validation studies must be performed to ensure the clinical applicability of our array-MLPA assay.

To improve the reliability of detection, we designed two different probes per targeted gene, located in the $5^{\prime}$ and 3' region of the same gene, and the average values of two probes were used to calculate the copy number of the gene. The data indicated that $89.4 \%$ of the relative probe signals from array-MLPA ranged from 0.85 to 1.15 in the normal controls. In the previous MLPA analysis [26], the normal region was usually set defined as 0.7 to 1.3 . This indicates that array-MLPA is more accurate than the reported results with conventional MLPA for the determination of copy number.

It is well-known that intrinsic sensitivity limitations of MLPA make it unsuitable for reliable detection of mosaicism. It has become possible to detect the mosaicism of chromosomal aneuploidies by array-MLPA due to its higher accuracy. In this study, we identified sex chromosome mosaicism in two patients by array-MLPA. In one case (B10), the average copy number on chromosome $\mathrm{X}$ was 0.71 revealing approximately $50 \%$ mosaic rate of chromosome $X$. The result was concordant with that of conventional cytogenetic analysis. In another case (B11), a similar result was observed on arrayMLPA. However, G-banded karyotype analysis indicated the chromosome karyotypes 45,X(70\%)/47, XXX(30\%). The result showed that there were polyploid cells in the patient B11. This suggests array-MLPA might have a bias in detection. Array-MLPA appeared to be more 
A

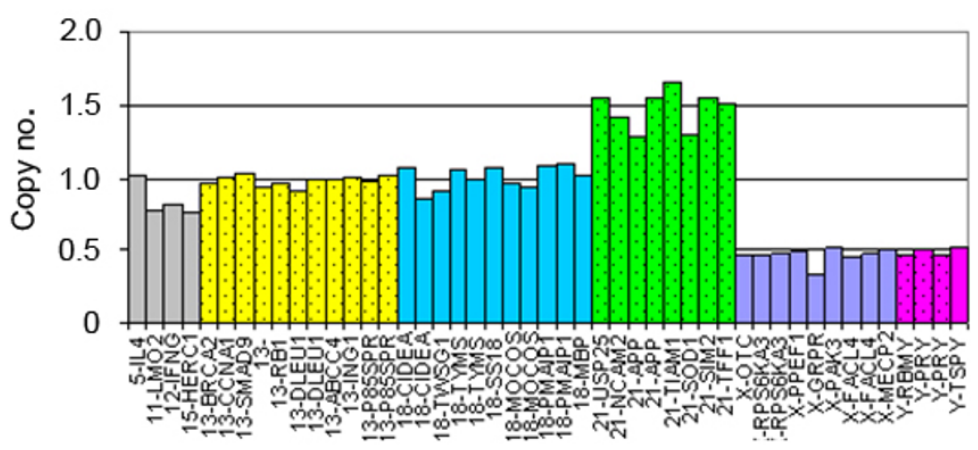

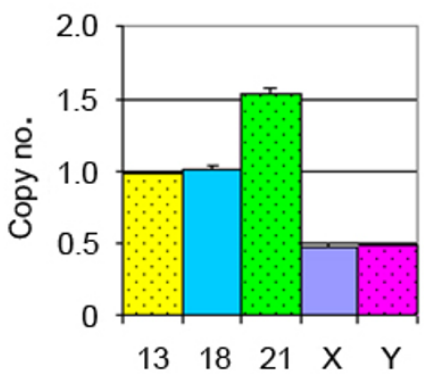

\begin{tabular}{|c|c|c|}
\hline Chromosome & Number of probes & A9 \\
\hline 13 & 11 & $0.99 \pm 0.01$ \\
\hline 18 & 11 & $1.02 \pm 0.02$ \\
\hline 21 & 8 & $1.53 \pm 0.05$ \\
\hline$x$ & 9 & $0.47 \pm 0.02$ \\
\hline Y & 4 & $0.49 \pm 0.01$ \\
\hline \multicolumn{2}{|c|}{ Description } & 21 Trisomy \\
\hline
\end{tabular}

B
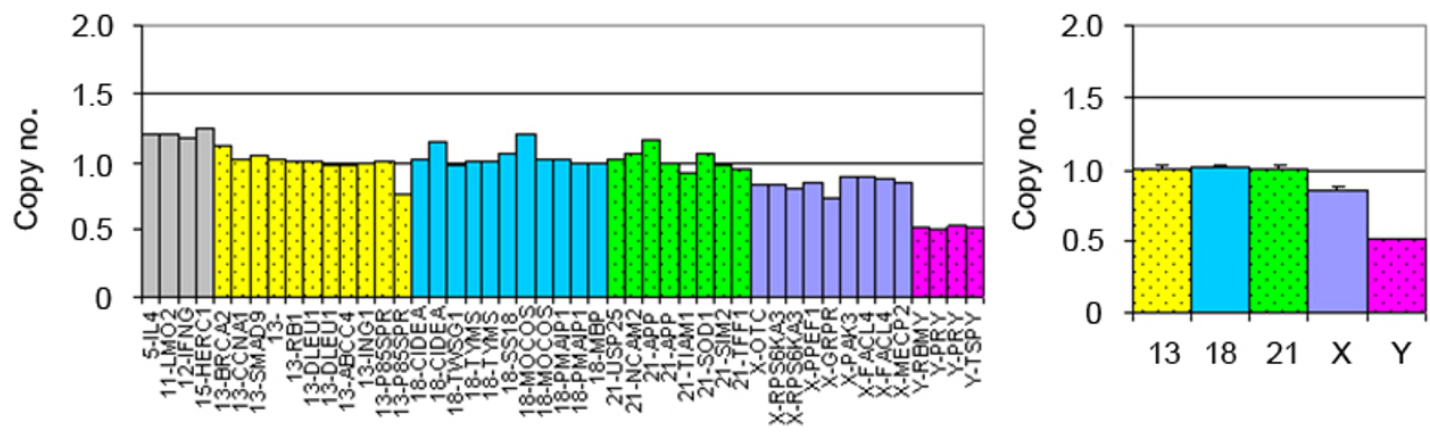

\begin{tabular}{ccc}
\hline Chromosome & Number of probes & $\mathbf{A 1 0}$ \\
13 & 11 & $1.00 \pm 0.03$ \\
18 & 11 & $1.01 \pm 0.02$ \\
21 & 8 & $1.01 \pm 0.03$ \\
$\mathrm{X}$ & 9 & $0.85 \pm 0.02$ \\
$\mathrm{Y}$ & 4 & $0.51 \pm 0.01$ \\
\hline \multicolumn{3}{c}{ Description } \\
\hline
\end{tabular}

Figure 3 The male fetus with trisomy 21 and XXY were analyzed with array-MLPA. The left figures showed the relative signal of each probe. The probes on chromosomes 13, 18, 21, X and $Y$ were depicted in yellow, blue, green, purple and purplish red, respectively. Eight autosomal control probes were shown in grey. The right figures showed the average copy numbers on each chromosome. Error bars represented the corresponding SD of the copy numbers on the MLPA probes covering each chromosome. The copy number of each chromosome was listed in the tables. 
Table 3 Comparison of the results from array-MLPA and karyotyping

\begin{tabular}{lcccc}
\hline & Blood & $\begin{array}{c}\text { Amniotic } \\
\text { fluid }\end{array}$ & Total & $\begin{array}{c}\text { Mismatch } \\
\text { genotype }\end{array}$ \\
\hline Normal control & 85 & 1 & 86 & $0 / 86(0 \%)$ \\
$\begin{array}{l}\text { Nonmosaic } \\
\text { aneuploidy }\end{array}$ & 69 & 11 & 80 & $4 / 80(5 \%)$ \\
Mosaic aneuploidy & 7 & 0 & 7 & $0 / 7(0 \%)$ \\
Total & 161 & 12 & 173 & $4 / 173(2.3 \%)$ \\
\hline
\end{tabular}

suitable for detecting mosaicism of monosomy than polyploidy.

In addition to $\mathrm{X}$ chromosome aneuploidies, unknown marker chromosomes in four cases (B5, B6, B8 and B9) were found by karyotype analysis, but G-banding did not distinguish their origins. Finally, we identified the regions of these unknown marker chromosomes by array-MLPA. Two of the four cases were confirmed by Y-chromosome-specific PCR. In another case (B7),

A
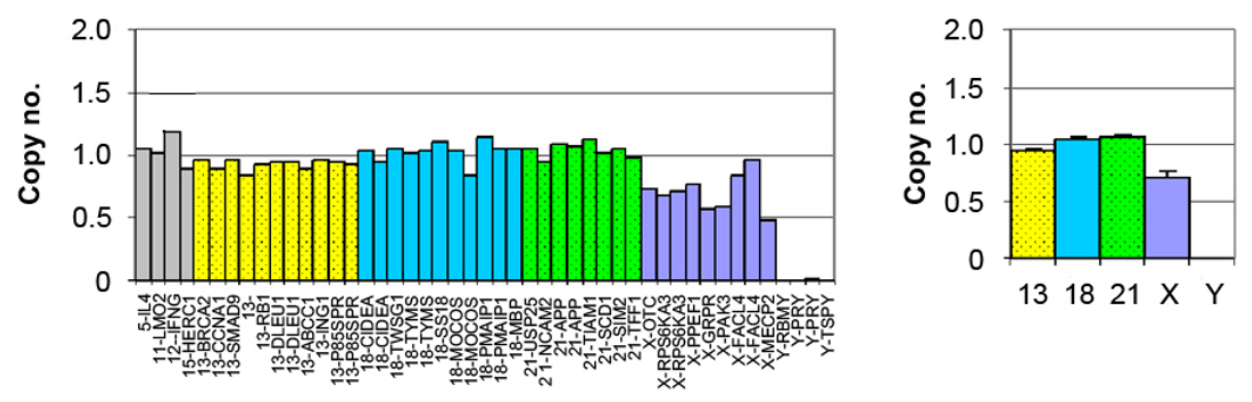

B

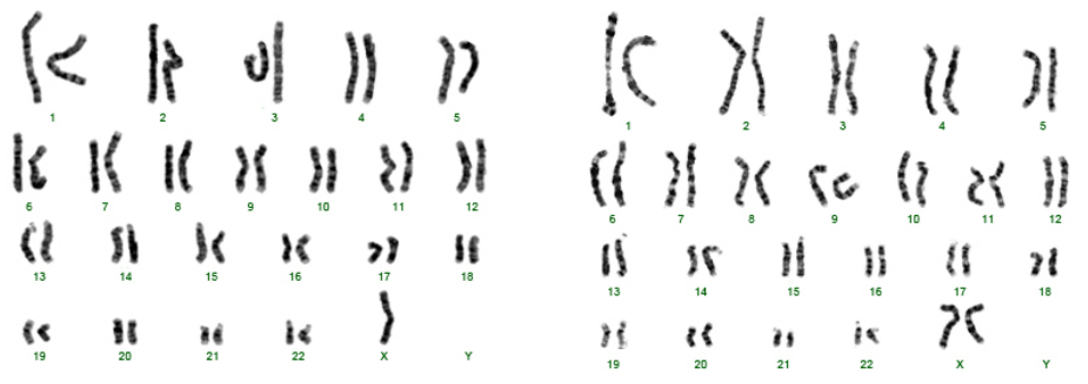

C
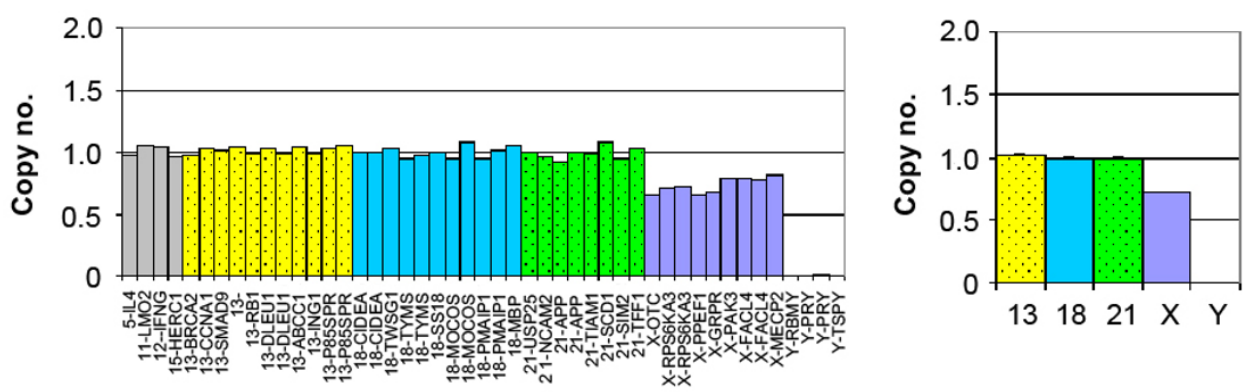

D

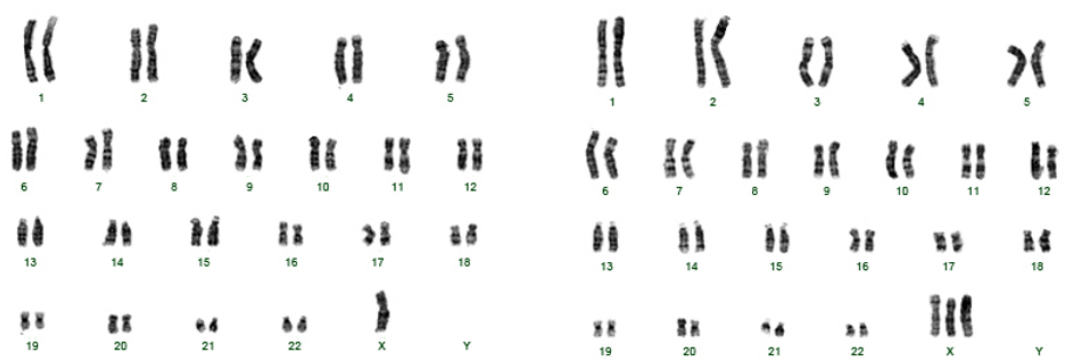

Figure 4 Array-MLPA analysis of chromosome $X$ monosomy mosaicism. (A) A female patient (B10) with mosaicism. The average copy number on chromosome $X$ was 0.71 . (B) G-banding analysis revealed the patient had the karyotype $45, X / 46, X, i(X)(q 10)$. (C) A female patient (B11) with mosaicism. The average copy number on chromosome $X$ was 0.72 . (D) G-banding analysis revealed the patient had the karyotype $45, X$ $(70 \%) / 47, X X X(30 \%)$. Error bars represented the corresponding SD of the copy numbers on the MLPA probes covering each chromosome. 


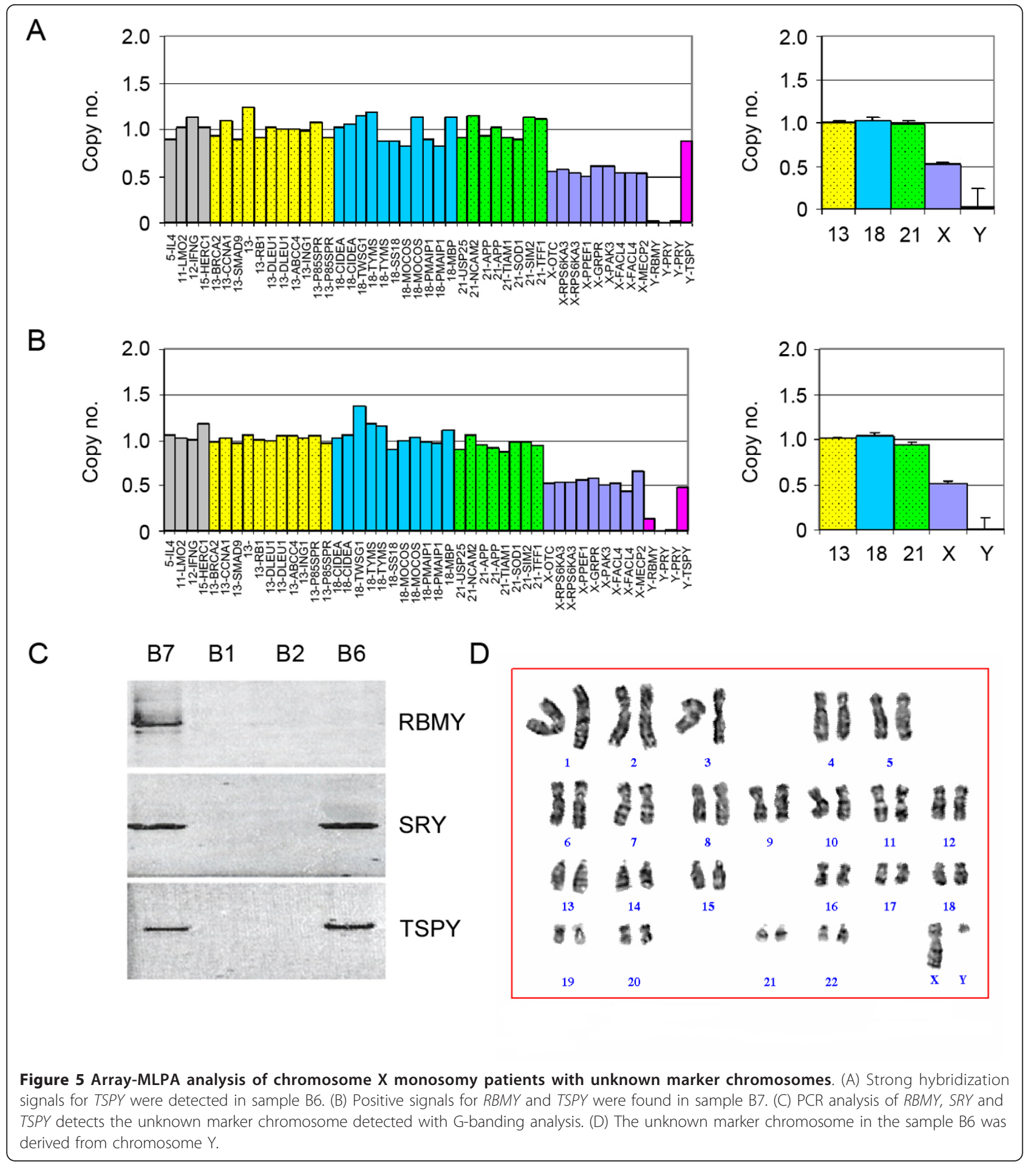

abnormality on the $\mathrm{Y}$ chromosome was identified by both array-MLPA and G-banding analysis. In this study, we designed ten pairs of probes to cover the long and short arms of chromosome Y. The data obtained on array-MLPA and PCR analysis indicate the presence of Y chromosome-specific probe signals, especially TSPY probes. Our study demonstrates the power of arrayMLPA for detecting small abnormalities on chromosomes.

However, four samples with chromosome translocations or inversions were not identified by array-MLPA since the recombination regions were not covered by 
the MLPA probes. Currently, we have only used one (Cy5) of the four available detection wavelengths for screening chromosomes 13, 18, 21, X, and Y. Using three additional sets of amplification primers, each labelled with a different fluorophore [32], it should be simple to expand the number of MLPA probes for other chromosomal abnormalities with potential clinical significance. Still, array-MLPA cannot completely replace conventional cytogenetic analysis. It is suitable to be used as a screening test in conjunction with karyotype analysis for diagnosis.

\section{Conclusions}

Our study demonstrates the successful application of array-MLPA for clinical molecular diagnosis with rapid and sensitive screening for chromosomal aneuploidies. Furthermore, we have developed a simple and rapid procedure for screening copy numbers on chromosomes $13,18,21, \mathrm{X}$, and Y using array-MLPA. Since array processing and data analysis are fully automated, array-MLPA should be suitable for large scale testing for chromosome aneuploidies in clinical diagnostic settings.

\section{Additional material}

Additional file 1: Table S1: Probe sequence for chromosomal
aneuploidy
Additional file 2: Figure S1: Schematic depiction of the array
technology. (A) Four-well array system was used in the study. Each array
contained 412 spots, and the oligonucleotide was immobilized on an
aluminum-oxide substrate. The substrate had a thickness of $60 \mu \mathrm{m}$ with
long capillary pores. The diameter of an individual pore was between
100 and 200 nm. A single spot occupied about 100,000 pores of the
substrate. (B) Raw image obtained on an array. These images were
recorded at 500 ms, 1000 ms, 1500 ms and 3000 ms exposure time
using a Cy5 filter set. This array design contained 120 probes spotted in
duplicate ( 240 spots), including 116 universal tag-probe oligonucleotides
for different chromosomes, two exogenous target ArrayControl RNA
Spikes (AM1780, Ambion, Austin, TX) oligonucleotides used as a negative
control for array hybridization, and two Cy5-reference oligonucleotides.
Additional file 3: Table S2: Gene copy numbers on array-MLPA in
normal controls

\section{Abbreviations}

MLPA: multiplex ligation-dependent probe amplification; FISH: fluorescence in situ hybridization; QF-PCR: Multiplex quantitative fluorescence PCR; CV: Coefficient of variation; ISCN: An international system for human cytogenetic nomenclature

\section{Acknowledgements}

The authors thank Dr Yi-Hua Guan for G-banding analysis. This work was financially supported by grants for the National Basic Research "973" Program of China (2007CB511904), National "863" Program of China (2007AA02Z400), Key Project from Shanghai Municipality (08JC1413000), Shanghai Shen-Kang Hospital Developmental Center (SHDC12007101), JointKey Project from Shanghai Health Bureau (2008ZD004), Shanghai Leading Academic Discipline (B204) and IS Pam-China Program from the Dutch SenterNovem (ISOM52003).

\section{Author details}

${ }^{1}$ Institute of Medical Genetics, Children's Hospital of Shanghai, Shanghai Jiao Tong University, Shanghai, P.R. China. Key Lab of Embryo Molecular Biology, Ministry of Health, and Shanghai Lab of Embryo and Reproduction Engineering, Shanghai, P.R. China. ${ }^{3}$ PamGene International BV, 'sHertogenbosch, The Netherlands. ${ }^{4}$ Medical Genetic Institute of Henan Province, the People's Hospital of Henan Province, Zhengzhou, P.R. China. ${ }^{5}$ Institute of Medical Sciences, Shanghai Jiao Tong University School of Medicine, Shanghai, P.R. China.

\section{Authors' contributions}

$J Y, Z R, Y-T W$ and SL were involved in patient and control subject recruitments. MM, RVB and $Y W$ participated in the design of MLPA probes and analysis software. JY, MX, CX, and DZ were involved in the array-MLPA experiments and data analysis. $Y H$ participated in the karyotyping analysis. $F Z, Y W$ and $Y Z$ conceived of the study, and participated in its design and coordination. JY, ZR, FZ and YW wrote the manuscript. All authors contributed to and have approved the final manuscript.

\section{Competing interests}

The authors declare that they have no competing interests.

Received: 31 January 2011 Accepted: 17 May 2011

Published: 17 May 2011

\section{References}

1. Lomax B, Tang S, Separovic E, Phillips D, Hillard E, Thomson T, Kalousek DK: Comparative genomic hybridization in combination with flow cytometry improves results of cytogenetic analysis of spontaneous abortions. Am J Hum Genet 2000, 66:1516-1521.

2. Yusuf RZ, Naeem R: Cytogenetic abnormalities in products of conception: A relationship revisited. Am J Reprod Immunol 2004, 52:88-96.

3. Hochstenbach R, Meijer J, van de Brug J, Vossebeld-Hoff I, Jansen R, van der Luijt RB, Sinke RJ, Page-Christiaens GC, Ploos van Amstel JK, de Pater JM: Rapid detection of chromosomal aneuploidies in uncultured amniocytes by multiplex ligation-dependent probe amplification (MLPA). Prenat Diagn 2005, 25:1032-1039.

4. Smith K, Lowther G, Maher E, Hourihan T, Wilkinson T, Wolstenholme J: The predictive value of findings of the common aneuploidies, trisomies 13, 18 and 21, and numerical sex chromosome abnormalities at CVS: experience from the ACC U.K. Collaborative Study. Association of Clinical Cytogeneticists Prenatal Diagnosis Working Party. Prenat Diagn 1999, 19:817-826.

5. Kuo WL, Tenjin H, Segraves R, Pinkel D, Golbus MS, Gray J: Detection of aneuploidy involving chromosomes 13,18 or 21 , by fluorescence in situ hybridization (FISH) to interphase and metaphase amniocytes. Am J Hum Genet 1991, 49:112-119.

6. Klinger $K$, Landes $G$, Shook D, Harvey $R$, Lopez L, Locke P, Lerner T, Osathanondh R, Leverone B, Houseal T, Pavelka K, Dackowski W: Rapid detection of chromosome aneuploidies in uncultured amniocytes by using fluorescence in situ hybridization (FISH). Am J Hum Genet 1992, 51:55-65.

7. Mansfield ES: Diagnosis of Down syndrome and other aneuploidies using quantitative polymerase chain reaction and small tandem repeat polymorphisms. Hum Mol Genet 1993, 2:43-50.

8. Ward BE, Gersen SL, Carelli MP, McGuire NM, Dackowski WR, Weinstein M, Sandlin C, Warren R, Klinger KW: Rapid prenatal diagnosis of chromosomal aneuplodies by fluorescence in situ hybridisation: clinical experience with 4500 specimens. Am J Hum Genet 1993, 52:854-865.

9. Philip J, Bryndorf B, Christensen B: Prenatal aneuploidy detection in interphase cells by fluorescence in siu hybridization (FISH). Prenat Diagn 1994, 14:1203-1215.

10. Adinolfi M, Sherlock J, Pertl B: Rapid detection of selected aneuploidies by quantitative fluorescent PCR. Bioessays 1995, 17:661-664.

11. Mann K, Fox SP, Abbs SJ, Yau SC, Scriven PN, Docherty Z, Ogilvie CM: Development and implementation of a new rapid aneuploidy diagnostic service within the UK National health service and implications for the future of prenatal diagnosis. Lancet 2001, 358:1057-1061.

12. Mann K, Donaghue C, Fox SP, Docherty Z, Ogilvie CM: Strategies for the rapid prenatal diagnosis of chromosome aneuploidy. Eur J Hum Genet 2004, 12:907-915. 
13. Tepperberg J, Pettenati MJ, Rao PN, Lese CM, Rita D, Wyandt H, Gersen S, White $\mathrm{B}$, Schoonmaker MM: Prenatal diagnosis using interphase fluorescence in situ hybridization (FISH): 2-year multi-center retrospective study and review of the literature. Prenat Diagn 2001, 21:293-301.

14. Witters I, Devriendt K, Legius E, Matthijs G, Van Schoubroeck D, Van Assche FA, Fryns JP: Rapid prenatal diagnosis of trisomy 21 in 5049 consecutive uncultured amniotic fluid samples by fluorescence in situ hybridisation (FISH). Prenat Diagn 2002, 22:29-33.

15. Ogilvie CM, Lashwood A, Chitty L, Waters JJ, Scriven PN, Flinter F: The future of prenatal diagnosis: rapid testing or full karyotype? An audit of chromosome abnormalities and pregnancy outcomes for women referred for Down's Syndrome testing. BJOG 2005, 112:1369-1375.

16. Cirigliano V, Ejarque M, Fuster C, Adinolfi M: X chromosome dosage by quantitative fluorescent $P C R$ and rapid prenatal diagnosis of sex chromosome aneuploidies. Mol Hum Reprod 2002, 8:1042-1045.

17. Cirigliano V, Voglino G, Cañadas MP, Marongiu A, Ejarque M, Ordoñez E, Plaja A, Massobrio M, Todros T, Fuster C, Campogrande M, Egozcue J, Adinolfi M: Rapid prenatal diagnosis of common chromosome aneuploidies by QF-PCR. Assessment on 18,000 consecutive clinical samples. Mol Hum Reprod 2004, 10:839-846.

18. Cirigliano V, Voglino G, Marongiu A, Cañadas P, Ordoñez E, Lloveras E, Plaja A, Fuster C, Adinolfi M: Rapid prenatal diagnosis by QF-PCR: evaluation of 30,000 consecutive clinical samples and future applications. Ann N Y Acad Sci 2006, 1075:288-298.

19. Waters JJ, Mann K, Grimsley L, Ogilvie CM, Donaghue C, Staples L, Hills A, Adams T, Wilson C: Complete discrepancy between QF-PCR analysis of uncultured villi and karyotyping of cultured cells in theprenatal diagnosis of trisomy 21 in three CVS. Prenat Diagn 2007, 27:332-339.

20. Schouten JP, McElgunn CJ, Waaijer R, Zwijnenburg D, Diepvens F, Pals G: Relative quantification of 40 nucleic acid sequences by multiplex ligation-dependent probe amplification. Nucleic Acids Res 2002, 30:e57.

21. Slater HR, Bruno DL, Ren H, Pertile M, Schouten JP, Choo KH: Rapid, high throughput prenatal detection of aneuploidy using a novel quantitative method (MLPA). J Med Genet 2003, 40:907-912.

22. Sellner LN, Taylor GR: MLPA and MAPH: new techniques for detection of gene deletions. Hum Mutat 2004, 23:413-419.

23. Ganesamoorthy D, Bruno DL, Schoumans J, Storey E, Delatycki MB, Zhu D, Wei MK, Nicholson GA, McKinlay Gardner RJ, Slater HR: Development of a multiplex ligation-dependent probe amplification assay for diagnosis and estimation of the frequency of spinocerebellar ataxia type 15. Clin Chem 2009, 55:1415-1418.

24. Gatta V, Scarciolla O, Gaspari AR, Palka C, De Angelis MV, Di Muzio A, Guanciali-Franchi P, Calabrese G, Uncini A, Stuppia L: Identification of deletions and duplications of the DMD gene in affected males and carrier females by multiple ligation probe amplification (MLPA). Hum Genet 2005, 117:92-98.

25. Lalic T, Vossen RH, Coffa J, Schouten JP, Guc-Scekic M, Radivojevic D, Djurisic M, Breuning MH, White SJ, den Dunnen JT: Deletion and duplication screening in the DMD gene using MLPA. Eur J Hum Genet 2005, 13:1231-1234.

26. van Opstal $D$, Boter $M$, de Jong $D$, van den Berg $C$, Brüggenwirth $H T$, Wildschut HI, de Klein A, Galjaard RJ: Rapid aneuploidy detection with multiplex ligation-dependent probe amplification: a prospective study of 4000 amniotic fluid samples. Eur J Hum Genet 2009, 17:112-121.

27. Zeng F, Ren ZR, Huang SZ, Kalf M, Mommersteeg M, Smit M, White $S$, Jin CL, Xu M, Zhou DW, Yan JB, Chen MJ, van Beuningen R, Huang SZ, den Dunnen J, Zeng YT, Wu Y: Array-MLPA: comprehensive detection of deletions and duplications and its application to DMD patients. Hum Mutat 2008, 29:190-197.

28. Shaffer $L G$, Slovak ML, Campbell LJ: An international system for human cytogenetic nomenclature. 2009, 16-31.

29. van Beuningen $R$, van Damme $H$, Boender $P$, Bastiaensen $N$, Chan $A$, Kievits T: Fast and specific hybridisation using flow-through microarrays on porous metal oxide. Clin Chem 2001, 47:1931-1933.

30. Wu Y, de Kievit P, Vahlkamp L, Pijnenburg D, Smit M, Dankers M, Melchers D, Stax M, Boender PJ, Ingham C, Bastiaensen N, de Wijn R, van Alewijk D, van Damme H, Raap AK, Chan AB, van Beuningen R: Quantitative assessment of a novel flow-through porous microarray for the rapid analysis of gene expression profiles. Nucleic Acids Res 2004, 32 e123.
31. Gibbons B, Datta P, Wu Y, Chan A, Armour JA: Microarray MAPH: accurate array-based detection of relative copy number in genomic DNA. BMC Genomics 2006, 7:163.

32. Harteveld CL, Voskamp A, Phylipsen M, Akkermans N, den Dunnen JT, White SJ, Giordano PC: Nine unknown rearrangements in 16p13.3 and 11 p15.4 causing alpha- and beta-thalassaemia characterised by high resolution multiplex ligation-dependent probe amplification. J Med Genet 2005, 42:922-931.

Pre-publication history

The pre-publication history for this paper can be accessed here: http://www.biomedcentral.com/1471-2350/12/68/prepub

doi:10.1186/1471-2350-12-68

Cite this article as: Yan et al:: Rapid screening for chromosomal aneuploidies using array-MLPA. BMC Medical Genetics 2011 12:68.

\section{Submit your next manuscript to BioMed Central and take full advantage of:}

- Convenient online submission

- Thorough peer review

- No space constraints or color figure charges

- Immediate publication on acceptance

- Inclusion in PubMed, CAS, Scopus and Google Scholar

- Research which is freely available for redistribution

Submit your manuscript at www.biomedcentral.com/submit
C) Biomed Central 